Regards sur l'économie allemande

Bulletin économique du CIRAC

$96 \mid 2010$

Varia

\title{
OHB Technology AG : une familiale la tête dans les étoiles mais les pieds sur terre
}

\section{Caroline Guiot}

\section{(2) OpenEdition}

\section{Journals}

Édition électronique

URL : http://journals.openedition.org/rea/4085

DOI : $10.4000 /$ rea. 4085

ISBN : 978-2-8218-0888-1

ISSN : 1965-0787

\section{Éditeur}

CIRAC

\section{Édition imprimée}

Date de publication : 1 mai 2010

Pagination : 33-38

ISSN : 1156-8992

\section{Référence électronique}

Caroline Guiot, "OHB Technology AG : une familiale la tête dans les étoiles mais les pieds sur terre », Regards sur l'économie allemande [En ligne], 96 | mai 2010, mis en ligne le 01 mai 2012, consulté le 19 avril 2019. URL : http://journals.openedition.org/rea/4085; DOI : 10.4000/rea.4085 


\section{OHB Technology AG : une familiale la tête dans les étoiles mais les pieds sur terre}

\section{Caroline Guiot}

Les bonnes nouvelles se bousculent en ce début d'année 2010 pour OHB : le 7 janvier, l'agence spatiale européenne (European Space Agency, ESA) a choisi le consortium (entités publiques et privées rassemblées pour réaliser un projet donné) emmené par sa filiale OHB System, pour la conception et la fabrication d'un premier lot de 14 satellites du système de navigation européen Galileo (futur concurrent de l'actuel GPS américain), contrat s'élevant à 566 millions $€$, soit deux fois le CA 2009 d'OHB, en compétition avec le géant Astrium (filiale d'EADS). Le 24 février, c'est l'agence spatiale allemande (Deutsches Zentrum für Luft- und Raumfahrt, DLR) qui confiait la Phase de définition (phase B) relative au programme DEOS (Deutsche Orbitale Servicing Mission) à OHB System. Le 19 mars, I'ESA a annoncé le début des négociations contractuelles avec le consortium Thalès-Alenia-Space/OHB System pour la construction de satellites météorologiques (Meteosat Third Generation, MTG), le préférant une nouvelle fois à la proposition d'Astrium.

Dans le même temps, et malgré la crise économique et financière mondiale, tous les indicateurs sont au vert: un chiffre d'affaires en hausse de $24 \%$ entre 2008 et 2009, s'établissant à 287 millions $€$, et une croissance du résultat courant avant impôt de $11 \%$, à 20,8 millions d'euros. Ces succès sont le résultat d'une conjugaison de facteurs : l'engagement visionnaire d'un homme dans le secteur spatial, scientifique et technologique, une gestion rigoureuse, entrepreneuriale et familiale toujours menée par la famille Fuchs, alors qu'OHB est la première société allemande du secteur spatial à être cotée en bourse, des relations nouées avec des partenaires tant institutionnels (DLR) que privés permettant de monter des consortiums pertinents pour répondre aux grands défis technologiques du secteur.

L'histoire d'OHB plonge ses racines dans celle de l'Allemagne de l'aprèsSeconde Guerre mondiale, avec les débuts de l'aventure aéronautique et spatiale allemande et européenne. Manfred Fuchs, né en 1938 dans le sud Tyrol (Italie), rêvait de posséder une compagnie aérienne. II a 17 ans en 1955 lorsqu'il part, son brevet de pilote en poche, faire des études d'ingénieur en aéronautique à Munich. A 21 ans, il rejoint Hambourg pour être mécanicien d'avion dans la société Hamburger Flugzeugbau GmbH (aujourd'hui Airbus). En juin 1961, celle-ci s'associe à la Weserflug und Focke-Wulf (WFW) basée à Brême pour créer une joint-venture : Entwicklungsring Nord (ERNO), dans laquelle est envoyé le jeune Manfred Fuchs, alors spécialiste en aérodynamique et mécanique. Quelques mois plus tard, il change de service et travaille au département spatial d'ERNO sur un projet de fusée-sonde, puis sur le projet de Spacelab (voir encadré ci-dessous). II s'initie ainsi aux projets d'envergure internationale, réalisés par des consortiums européens d'entreprises et d'institutions publiques de recherche. En décembre 1982, M. Fuchs développe avec deux autres partenaires le concept de la station spatiale européenne Columbus. De chef de projet, il deviendra directeur de division, avant de quitter l'entreprise en 1985. 
En 1945, les sciences spatiales ont été interdites en Allemagne, au même titre que la R\&D militaire. Les chercheurs exilés ne sont revenus en Allemagne que dix ans plus tard, lorsque les champs d'études aérospatiales, militaires et nucléaires ont de nouveau été autorisés. Pendant ce temps, les grands groupes allemands œuvrant dans l'aéronautique se sont tournés vers d'autres domaines d'activité, comme Dornier dans l'industrie textile, avant de pouvoir de nouveau en 1954 revenir à leur cœur de métier aéronautique et militaire. C'est à cette date que la Communauté allemande de la recherche (Deutsche Forschungsgemeinschaft, DFG) a pu financer la recherche spatiale. En 1960, cet organisme a participé à la réflexion sur les activités spatiales au niveau européen, et à la fondation de l'ELDO (European Launcher Development Organization) et de l'ESRO (European Space Research Organization). Le premier satellite allemand a été lancé en 1969, année de la création de l'Institut allemand de test et de recherche pour l'aviation et le spatial (Deutsche Forschungs- und Versuchanstalt für Luft- und Raumfahrt, DFVLR), ancêtre de l'actuelle DLR.

Les trois plus grandes entreprises du secteur de cette époque : MBB - Messerschmitt-Bölkow-Blohm (aujourd'hui intégrée dans EADS), Dornier et ERNO (Entwicklungsring Nord, une joint-venture entre deux sociétés d'aéronautique de Brême et de Hambourg), s'engagent dans les programmes de recherche coordonnés par l'agence spatiale européenne (ESA), créée en 1975. L'Allemagne prend ainsi le leadership pour la création du Spacelab (laboratoire à microgravité modulaire destiné à être envoyé dans l'espace grâce à une navette spatiale américaine), alors que la France se concentre sur le programme de la fusée Ariane. Le Spacelab n'a pas porté tous les fruits escomptés au niveau européen, car le module a été donné à la NASA afin que les spationautes européens puissent avoir des places dans les vols habités. Cependant, les industriels ayant participé à ce programme ont acquis des savoir-faire importants sur ces techniques de conception et de fabrication de modules spatiaux. Outre le rayonnement scientifique international, l'Allemagne souligne, dès les débuts, l'importance des investissements industriels liés aux programmes spatiaux pour développer un secteur d'activité nouveau et plein de promesses de progrès technologiques.

Parallèlement, Mme Christa Fuchs, née à Hambourg en 1938, épouse de Manfred Fuchs, est gérante. Installée à Brême avec son mari, elle reprend en 1982, après que ses deux enfants (Marco et Romana) ont fini leurs études, une petite entreprise créée en 1958 par la famille Otto (qui n'avait pas d'héritier à qui la transmettre) : la SARL OHB - Otto Hydrolik Bremen $\mathrm{GmbH}$, un atelier de réparation électronique et hydraulique pour les bateaux des forces navales allemandes, employant 5 personnes. En 1985, Manfred Fuchs quitte ERNO pour $\mathrm{OHB}$, apportant ses connaissances et savoir-faire dans le domaine du spatial.

\footnotetext{
Quelques dates clefs

1958 : Création de la SARL OHB - Otto Hydrolik Bremen GmbH.

1982: Christa Fuchs acquiert OHB.

1985: Manfred Fuchs entre dans la société : c'est le virage vers le spatial.

1991: OHB change de dénomination tout en conservant le même acronyme : Orbital- und Hydrotechnologie BremenSystem $\mathrm{GmbH}$.

1993 : Création de la société OHB Teledata $\mathrm{GmbH}$ (Brême), filiale d'OHB.

2000: La dénomination se précise et s'affirme : OHB - Orbitale Hochtechnologie Bremen System GmbH.

2001: Introduction en bourse de la filiale OHB Teledata (nouveau marché, place de Francfort). OHB prend une participation dans le capital de ORBCOMM (USA) et négocie une exclusivité de distribution du service en Europe. Le consortium emmené par OHB System remporte le projet SAR-Lupe : conception et construction d'une constellation de 5 satellites radar pour l'armée allemande.

2002: Regroupement de OHB System et OHB Teledata au sein du groupe OHB Technology AG (Brême), dont les actions sont cotées. Ces deux sociétés forment les deux divisions historiques du groupe.

2004: OHB System remporte un contrat pour la livraison et le lancement de 6 satellites ORBCOMM.

2005: OHB acquiert la société MAN-Technologie AG, filiale «spatial » du groupe d'ingénierie MAN, qui, renommée MT Aerospace AG (Augsbourg), forme la division Space Transportation + Aerospace Structures de OHB Technology AG.

2007: OHB acquiert la société Kayser-Threde $\mathrm{GmbH}$ (Munich), troisième plus importante société de technologie spatiale en Allemagne. Cette activité forme la division Payloads + Science.

2008: Année des lancements réussis de plusieurs satellites, dont les programmes s'échelonnent sur plusieurs années : les 6 satellites ORBCOMM sont lancés avec succès; de même le $5^{\mathrm{e}}$ et dernier satellite SAR-Lupe ; I'ATV (Automatic Transfer Vehicle) rejoint la Station Spatiale Internationale, tout comme le module Columbus.

2009: OHB acquiert la société italienne Carlo Gavazzi Space Spa, dans laquelle elle avait déjà une participation depuis 1995, et renforce la présence internationale de OHB.
}

La croissance de l'entreprise suit celle des projets et des rapprochements stratégiques avec des sociétés avec lesquelles OHB collabore. Ainsi, si le chiffre d'affaires augmente de façon très régulière, les paliers de croissance correspondent à l'élargissement du périmètre d'OHB : en 2005, l'intégration de la filiale « spatial » du groupe MAN-Technologie AG entraine une augmentation de plus de 400 personnes de l'effectif. Le même phénomène se répète en 2007 et en 2009. 
OHB Technology AG : un groupe en forte croissance

\begin{tabular}{|c|rr|rr|rr|}
\hline Année & CA (en K€) & Evol. & EBIT (en K€) & Evol. & Salariés & Evol. \\
$\mathbf{2 0 0 1}$ & 12439 & $N / A$ & 452 & $N / A$ & 125 & $N / A$ \\
$\mathbf{2 0 0 2}$ & 67961 & $446 \%$ & 3706 & $720 \%$ & 292 & $134 \%$ \\
$\mathbf{2 0 0 3}$ & 96349 & $42 \%$ & 6289 & $70 \%$ & 287 & $-2 \%$ \\
$\mathbf{2 0 0 4}$ & 137909 & $43 \%$ & 8467 & $35 \%$ & 282 & $-2 \%$ \\
$\mathbf{2 0 0 5}$ & 113829 & $-17 \%$ & 14080 & $66 \%$ & 795 & $182 \%$ \\
$\mathbf{2 0 0 6}$ & 163147 & $43 \%$ & 20428 & $45 \%$ & 823 & $4 \%$ \\
$\mathbf{2 0 0 7}$ & 218801 & $34 \%$ & 17486 & $-14 \%$ & 1189 & $44 \%$ \\
$\mathbf{2 0 0 8}$ & 232473 & $6 \%$ & 18708 & $7 \%$ & 1284 & $8 \%$ \\
$\mathbf{2 0 0 9}$ & 287164 & $24 \%$ & 20771 & $11 \%$ & 1546 & $20 \%$ \\
\hline
\end{tabular}

Source : Rapports annuels 2002 à 2009.

\section{OHB Technology AG : quatre divisions et une branche internationale}

OHB est aujourd'hui un petit groupe de 1546 salariés dont l'activité est répartie en 4 divisions (5 avec la branche internationale) conduites chacune par une société à identité forte.

OHB Technology AG : quatre divisions et une branche internationale

\begin{tabular}{|c|c|c|c|c|c|c|}
\hline Divisions & $\begin{array}{c}\text { CA } 2009 \\
\text { (en K€) }\end{array}$ & $\begin{array}{c}\text { Part (\%) } \\
\text { hors Holding } \\
\text { + Conso. }\end{array}$ & $\begin{array}{c}\text { EBIT } 2009 \\
\text { (en K€) }\end{array}$ & $\begin{array}{c}\text { Part (\%) } \\
\text { hors Holding } \\
\text { + Conso. }\end{array}$ & Salariés & $\begin{array}{c}\text { Part (\%) } \\
\text { hors Holding } \\
\text { + Conso. }\end{array}$ \\
\hline Space Systems + Security & 88727 & $29 \%$ & 3440 & $15 \%$ & 280 & $18 \%$ \\
\hline Payloads + Science & 61959 & $20 \%$ & 4094 & $18 \%$ & 227 & $15 \%$ \\
\hline Space International & 17696 & $6 \%$ & 3735 & $17 \%$ & 241 & $16 \%$ \\
\hline Space Transportation + Aerospace Structures & 123176 & $40 \%$ & 10637 & $47 \%$ & 704 & $46 \%$ \\
\hline Telematics + Satellite Operations & 17067 & $6 \%$ & 708 & $3 \%$ & 88 & $6 \%$ \\
\hline Holding + Consolidation & -21461 & $N / A$ & -1843 & $N / A$ & 6 & $N / A$ \\
\hline TOTAL & 287164 & $100 \%$ & 20771 & $100 \%$ & 1546 & $100 \%$ \\
\hline
\end{tabular}

Source : Rapport annuel 2009.

La division-phare de ce début d'année 2010 est la division historique du groupe OHB : Space System + Security. Les succès d'aujourd'hui (29 \% du CA 2009, mais $55 \%$ du carnet de commandes 2009, à quoi s'ajoute le contrat ESA Galileo) ont été construits depuis de nombreuses années. OHB System a ainsi développé depuis plus de 10 ans une véritable expertise dans le segment des petits satellites, en orbite basse (500 kilomètres au-dessus de la Terre) servant notamment pour la télédétection et les stations spatiales, ou en orbite géostationnaire (altitude $35784 \mathrm{~km}$, fixe à l'équateur) idéale pour les satellites de télécommunications et d'observation de la Terre. La première victoire d'envergure de OHB a été la signature en 2001 du contrat SAR-Lupe : une constellation de cinq satellites d'imagerie radar très haute résolution en orbite basse, à destination du Ministère allemand de la Défense (Bundesministerium der Verteidigung, BMVg, et Bundesamt für Wehrtechnik und Beschaffung, BWB). Ceux-ci ont été mis sur orbite avec succès entre 2006 et 2008 . Pour les dirigeants d'OHB, cette expérience pleinement réussie a été capitalisée et a servi de référence au moment du choix de l'attribution du contrat des satellites Galileo à OHB par l'ESA (entre autres atouts).

Parallèlement, le projet SmallGEO/Luxor développe une plateforme pour petits satellites géostationnaires pour la DLR, et pour l'ESA dans le cadre du programme ARTES-11; les programmes européens et canadiens Advanced Research in Telecommunications Systems (ARTES) sont destinés à financer et promouvoir les activités innovantes dans le domaine des produits et services liés aux satellites de communication. La division Space System + Security a encore deux autres activités. D'une part les vols habités: OHB est impliqué dans la conception et la réalisation du laboratoire spatial du module européen

Division Space System + Security 
Division
Payloads + Science

Division

Space Transportation

+ Aerospace Structures

Division

Telematics + Satellite Operations

Division
Space International

Partenariats privés : synergies et optimisation de l'expertise
Columbus de la Station Spatiale Internationale (ISS) ; d'autre part, les missions d'exploration : OHB participe à l'étude (phase 0) de la mission LEO (Lunar Exploration Orbiter) dont le but est de cartographier la Lune en haute définition et d'analyser ses propriétés physiques, ainsi qu'au programme ExoMars de l'ESA.

La division Payloads + Science a été formée suite au rachat par OHB de la société Kayser-Threde $\mathrm{GmbH}$. En 2009, elle représente $20 \%$ du CA. Son activité principale est le développement et la réalisation des "charges utiles », équipements et matériels pour les missions spatiales, vols habités (ISS) ou non. L'une des spécialités est le développement d'instruments de recherche en condition de microgravité. Un autre segment est relatif aux applications industrielles. Ainsi la technologie de contrôle des process (process control technology) permet de coupler des systèmes de centres de contrôle pour la Deutsche Bahn. Par ailleurs, le système embarqué d'enregistrement de données (MINIDAU) conçu par Kayser-Threde équipe aujourd'hui $70 \%$ des installations de tests de collision.

La division Space Transportation + Aerospace Structures, née de l'intégration de la société MT Aerospace, est centrée sur la fourniture de composants pour l'aéronautique et l'aviation, et les systèmes d'antennes. Avec cette activité, OHB s'affiche comme le plus important fournisseur allemand du programme Ariane 5 . En 2009, un contrat pour la fabrication d'éléments de 35 transporteurs Ariane supplémentaires a été signé. Par ailleurs, OHB joue un rôle crucial dans la production et l'assemblage des containers d'eau et de déchets pour Airbus. Enfin, le segment des antennes et de la mécatronique est assuré par la société MT Mechatronics $\mathrm{GmbH}$ (Mayence), active tant au niveau médical (traitement par radiations ionisantes) qu'en termes de construction de radio-télescopes, tel que le projet ALMA (Atacama Large Millimeter Array) US/Japon/Europe comprenant 64 antennes individuelles de 12 mètres de diamètre situées dans le désert de l'Atacama au Chili. Au total, cette division représente $40 \%$ du CA 2009 et est la plus rentable du groupe $\mathrm{OHB}$, générant $47 \%$ du résultat courant avant impôt. Les commandes engrangées au 31 décembre 2009 s'élèvent à 439,1 millions $€(31 \%)$.

L'autre division historique du groupe, Telematics + Satellite Operations, offre une large gamme de solutions embarquées et d'ordinateurs de bord pour le management des activités de transport (équipement de flottes de camions par exemple) via satellites : systèmes de navigation, de localisation, de communication, d'identification (scanner, RFID, etc.), ainsi que de solutions mobiles spécialisées. Toutes ces applications sont utilisées tant à des fins civiles et commerciales que militaires. De plus, les systèmes de télématique et de gestion des flux sont développés pour des sociétés de logistique, comme par exemple le projet « Yellow Box » pour DHL, présenté en 2009. L'autre activité de cette division offre les services de communication via satellites. Ainsi, sur la base des participations et des expertises acquises sur le système satellite ORBCOMM basé aux Etats-Unis, OHB bénéficie d'une exclusivité de distribution des services satellites sur le territoire européen via sa filiale ORBCOMM Deutschland fondée en 1998.

Enfin, une division Space International regroupe les sociétés œuvrant dans le domaine du spatial et ayant leur siège hors d'Allemagne. Le fait marquant en 2009 a été le rachat total du capital de Carlo Gavazzi Space Spa, une société italienne dans laquelle OHB avait pris une participation dès 1995 et qui a souvent été partenaire dans les consortiums emmenés par OHB pour les grands projets spatiaux (ex. SAR-Lupe).

\section{Une stratégie établie sur un réseau de partenariats}

Pour atteindre ses objectifs ambitieux et emporter les appels d'offres, OHB a choisi la voie des partenariats plutôt que celle de l'intégration complète, construisant des alliances industrielles aux expertises reconnues, spécifiques à chaque nouveau projet. Ainsi pour le projet SAR-Lupe, OHB s'est associé à 10 sociétés ou institutions: Thalès Alenia Space Toulouse, Carlo Gavazzi Space, 
COSMOS International, DLR GSOC (German Space Operation Center de l'agence spatiale allemande), EADS Defence \& Security, Rosoboronexport, RST (Radar Systemtechnik), RTG, Saab Space, Tesat Spacecom. Pour le projet Galileo signé début 2010, OHB s'est allié avec SSTL (Surrey Satellite Technology Ltd.) : OHB assure la coordination du projet et fournit les plateformes satellites, pendant que SSTL produit les charges utiles pour les satellites. Et aujourd'hui comme hier, alors que la compétition est de plus en plus âpre entre OHB et Astrium sur les grands projets européens, OHB continue de collaborer avec son rival dans certains projets. Pour autant, le président d'OHB reconnaît qu'il est plus aisé de construire un consortium avec Thalès Alenia Space qui est très peu présent en Allemagne.

Parallèlement, la mise en place, dès les débuts de l'entreprise, de collaborations avec la recherche plus institutionnelle, avec la DLR ou l'Université de Brême, ont permis à OHB de s'appuyer sur technologies de pointe, des brevets, des ingénieurs experts et des techniques innovantes. Les modalités de collaboration consistent soit dans l'intégration de l'institution au sein d'un consortium, l'entité recevant alors une part de financement pour développer une partie du projet, soit en agissant comme client privilégié, ainsi la DLR (Cologne) qui représente le principal client pour la technologie spatiale allemande. Les projets de long terme ainsi financés permettent le développement d'expertises spécifiques au sein des équipes $O H B$.

De même, les liens avec le ZARM (Zentrum für angewandte Raumfahrttechnologie und Mikrogravitation, Centre allemand des applications de la microgravité), institut de recherche appliquée au sein de l'Université de Brême, sont très étroits : le ZARM a en effet été initié par M. Fuchs, et OHB a largement participé au financement de la construction de la tour de chute libre, permettant le test de matériels en situation d'apesanteur. Le centre est présidé par le Prof. Hans J. Rath, ingénieur, qui est également membre du Conseil de surveillance d'OHB depuis 2001. En reconnaissance de ces liens étroits et soutiens autant matériels qu'immatériels, Christa et Manfred Fuchs ont été nommés conjointement membres d'honneur et bienfaiteurs de l'Université de Brême lors d'une cérémonie officielle à l'hôtel de ville de Brême le 27 août 2009.

Au sein du groupe, les rôles ont été définis dès les débuts d'OHB, lors de la reprise du petit atelier: Christa Fuchs est la gestionnaire de l'entreprise, quand Manfred Fuchs est l'ingénieur aéronautique, passionné de haute technologie spatiale. Leur fils, Marco Fuchs, après avoir exercé le métier d'avocat, est entré dans l'entreprise pour en prendre pas à pas la direction. Artisan de l'introduction en bourse de la société OHB Teledata et des rachats et prises de participation stratégiques, il a succédé à son père à la tête du directoire en 2001, Manfred Fuchs restant à ses côtés au sein du directoire, interlocuteur privilégié pour les questions scientifiques et stratégiques, pendant que Christa Fuchs préside le conseil de surveillance. Cette gestion familiale est une des forces d'OHB.

Et si le groupe est coté à la bourse de Francfort depuis 2001 (indice principal DAX, ainsi qu'au GEX - German Entrepreneurial Index), la famille Fuchs se réserve encore $69,72 \%$ du capital, gage d'une indépendance vis-à-vis des aléas des marchés financiers, comme d'une vision stratégique se développant sur le long terme. C'est là une caractéristique foncière des entreprises familiales, régulièrement soulignée par les études relatives à ces sociétés qui constituent $95,1 \%$ des entreprises allemandes - et appartiennent le plus souvent à la catégorie des PME.

Autant que la culture de partenariat et la gestion familiale, l'organisation mise en place au sein d'OHB est un facteur clé de succès et tient en deux mots: organigramme plat. Si c'est là une caractéristique foncière du mode de gouvernance des entreprises allemandes qui repose largement sur les responsabilités (compétences) individuelles, l'organigramme d'OHB est simplifié à l'extrême. Seuls trois niveaux hiérarchiques sont en effet recensés: le management
Partenariats de long terme avec la recherche ...

... et forte implication dans I'Université de Brême

La gestion d'OHB reste aux mains de la famille...

... bien que le groupe soit coté en bourse

Un organigramme plat favorable à l'innovation 
Un groupe qui recrute

OHB est un atout majeur du cluster Espace du Land de Brême board, le management par projet, puis les ingénieurs, ces derniers ayant un accès direct au management board. Une telle organisation facilite la circulation des informations et des idées, en allégeant considérablement les modalités de prises de décision, ainsi que la remontée des problèmes qui peuvent être résolus plus simplement. La réactivité induite par cette organisation est un atout majeur, de même que les coûts salariaux réduits, grâce à un poids bien moindre des charges indirectes. Plus important encore: elle crée le terreau favorable à la créativité des collaborateurs, autrement dit, à leur aptitude à innover en permanence, puisqu'elle valorise la responsabilité individuelle.

Tous ces éléments font la force d'OHB, tant en interne qu'au moment des propositions sur des projets d'envergure. Et ce sont les mêmes éléments qui sont mis en avant pour recruter les futurs collaborateurs. Les grands projets sélectionnés en ce début d'année 2010 entraînent une intensification du recrutement, essentiellement au sein de la société OHB System, mais aussi chez KayserThrede. Tous les profils sont proposés (plus d'une soixantaine de postes actuellement) : de l'assembleur de modules satellites aux chefs de projet, en passant par les responsables qualité, les ingénieurs en système de contrôle des altitudes et orbites, développeurs de logiciels spécifiques, etc.

Dans ce contexte porteur où d'importants contrats ont été signés, gages d'une activité intense pour les prochaines années, OHB peut s'appuyer sur le dynamisme de la ville-Etat de Brême. Plus petit des 16 Länder de RFA, il use de tous les réseaux et rouages pour mettre sur le devant de la scène ses segments innovants et technologies de pointe, et notamment le cluster Espace. La ville libre hanséatique est ainsi membre de plusieurs réseaux européens : ERAStar Regions, mettant en commun les moyens des régions ou petits pays européens dans le but de monter des coopérations de branche autour de programmes, notamment Galileo ou GMES (Global Monitoring for Environment and Security), NEREUS (Network for Regions Using Space Technologies), ERRIN (European Regions Research and Innovation Network), etc. Par ailleurs, la ville-Etat entend promouvoir l'innovation, soutenant deux programmes ambitieux : InnoVision 2010 et Science Plan 2010, dont l'un des secteurs majeurs est celui du spatial et de l'aéronautique.

MALGRÉ UN DÉBUT 2010 ÉCONOMIQUEMENT TRĖS POSITIF pour la société OHB, le défi majeur des années futures sera de rester fidèle aux principes qui fondent son identité et constituent sa force, malgré la concentration croissante dans le secteur du spatial et la multiplication des échéances (lancement du premier satellite Galileo prévu pour 2012, et livraison d'un nouvel exemplaire tous les mois et demi jusqu'en 2014). Car rien n'est jamais acquis, et OHB sera remis en concurrence pour le prochain lot de 8 satellites Galileo, face au rival Astrium, quand par ailleurs, des incertitudes planent actuellement sur le devenir de la constellation des satellites Galileo, dont la Chine dispute âprement les zones de fréquences, conflit en cours de négociation depuis plusieurs années et non encore résolu à ce jour...

\section{Indications bibliographiques}

\footnotetext{
- BouRgeols I., «Entreprises familiales: un rôle clef Outre-Rhin », Regards sur l'Economie Allemande, $\mathrm{n}^{\circ} 82 / 2007$

- FidDELKe T., « Ein Multitalent der Luft- und Raumfahrttechnik », Die Welt, 12-07-2001

- SchütTe G., « Innovation Policy in Bremen : Interlinkage of economic Policy and science Policy », PRIME Stakeholder Day on Regional Research and Innovation Policies, Paris, 10-02-2006

- WeYER J., « European Star Wars: The emergence of space technology through the interaction of military and civilian interest-groups », in Science Technology and the Military, Vol. XII, 1988, p.243-288

- www.bremen.de ; www.dlr.de ; www.era-star.net ; www.errin.eu ; www.esa.int ; www.ifm-bonn.org ; www.ohb-technology.de ; www.zarm.uni-bremen.de.
} 